\title{
In Vivo Microdialysis Reveals Age-Dependent Decrease of Brain Interstitial Fluid Tau Levels in P301S Human Tau Transgenic Mice
}

\author{
Kaoru Yamada, ${ }^{1}$ John R. Cirrito, ${ }^{1}$ Floy R. Stewart, ${ }^{1}$ Hong Jiang, ${ }^{1}$ Mary Beth Finn, ${ }^{1}$ Brandon B. Holmes, ${ }^{1}$ \\ Lester I. Binder, ${ }^{2}$ Eva-Maria Mandelkow, ${ }^{3}$ Marc I. Diamond, ${ }^{1}$ Virginia M.-Y. Lee, ${ }^{4}$ and David M. Holtzman ${ }^{1}$ \\ ${ }^{1}$ Department of Neurology, Hope Center for Neurological Disorders, Alzheimer's Disease Research Center, Washington University, St. Louis, Missouri \\ 63110, ${ }^{2}$ Department of Cell and Molecular Biology, Feinberg School of Medicine, Northwestern University, Chicago, Illinois 60611, ${ }^{3}$ Max Planck Unit for \\ Structural Molecular Biology, c/o DESY, 22607 Hamburg, Germany, and ${ }^{4}$ Center for Neurodegenerative Disease Research, Department of Pathology and \\ Laboratory Medicine, University of Pennsylvania School of Medicine, Philadelphia, Pennsylvania 19104
}

Although tau is a cytoplasmic protein, it is also found in brain extracellular fluids, e.g., CSF. Recent findings suggest that aggregated tau can be transferred between cells and extracellular tau aggregates might mediate spread of tau pathology. Despite these data, details of whether tau is normally released into the brain interstitial fluid (ISF), its concentration in ISF in relation to CSF, and whether ISF tau is influenced by its aggregation are unknown. To address these issues, we developed a microdialysis technique to analyze monomeric ISF tau levels within the hippocampus of awake, freely moving mice. We detected tau in ISF of wild-type mice, suggesting that tau is released in the absence of neurodegeneration. ISF tau was significantly higher than CSF tau and their concentrations were not significantly correlated. Using P301S human tau transgenic mice (P301S tg mice), we found that ISF tau is fivefold higher than endogenous murine tau, consistent with its elevated levels of expression. However, following the onset of tau aggregation, monomeric ISF tau decreased markedly. Biochemical analysis demonstrated that soluble tau in brain homogenates decreased along with the deposition of insoluble tau. Tau fibrils injected into the hippocampus decreased ISF tau, suggesting that extracellular tau is in equilibrium with extracellular or intracellular tau aggregates. This technique should facilitate further studies of tau secretion, spread of tau pathology, the effects of different disease states on ISF tau, and the efficacy of experimental treatments.

\section{Introduction}

Neurofibrillary tangles (NFTs) consist of fibrillar tau aggregates. They are a neuropathological hallmark of tauopathies including Alzheimer's disease (AD) and forms of frontotemporal dementia (FTD). Tau is normally a highly soluble, cytoplasmic protein. However, under pathological conditions, it is hyperphosphorylated and aggregates into filamentous structures. The NFT burden and distribution correlate well with cognitive decline in $\mathrm{AD}$ as well as in mouse models of tauopathy (Arriagada et al., 1992; Bancher et al., 1993; Small and Duff, 2008; Polydoro et al., 2009), and mutations in tau cause autosomal dominant forms of FTD (Ballatore et al., 2007). This strongly suggests that tau aggregation

Received May 23, 2011; revised July 2, 2011; accepted July 28, 2011.

Author contributions: K.Y., J.R.C., and D.M.H. designed research; K.Y., F.R.S., H.J., M.B.F., and B.B.H. performed research; H.J., B.B.H., L.I.B., E.-M.M., M.I.D., and V.M.-Y.L. contributed unpublished reagents/analytic tools; K.Y., J.R.C., F.R.S., M.B.F., and D.M.H. analyzed data; K.Y., M.I.D., V.M.-Y.L., and D.M.H. wrote the paper.

This work was supported by funding from AstraZeneca (D.M.H.), Cure Alzheimer's Fund (D.M.H.), Astellas Foundation for Research on Metabolic Disorders (K.Y.), the Uehera Memorial Foundation (K.Y.), and Japan Society for the Promotion of Science (K.Y.).

The authors declare no competing financial interests.

Correspondence should be addressed to David M. Holtzman, Department of Neurology, Washington University, 425 South Euclid Avenue, BJCIH ninth floor, Campus Box 8111, St. Louis, MO 63110. E-mail: holtzman@neuro.wustl.edu.

DOI:10.1523/JNEUROSCI.2569-11.2011

Copyright $\odot 2011$ the authors $\quad 0270-6474 / 11 / 3113110-08 \$ 15.00 / 0$ plays a key role in the progression of several neurodegenerative diseases (Lee et al., 2001).

Although tau is a cytoplasmic protein, it is also present in the CSF. Thus, tau is probably released from cells as a physiological process. CSF tau levels change under certain pathological conditions. For example, tau is increased after stroke (Hesse et al., 2001), markedly increased in prion diseases (Otto et al., 1997), and increased moderately in AD (Riemenschneider et al., 2003). Interestingly, however, in forms of FTD caused by tau mutations, CSF tau is not increased (Grossman et al., 2005). Interstitial fluid (ISF) tau has not been measured in animals, and its relationship to CSF tau is unknown. In addition to soluble tau that reaches the extracellular space, recent studies have shown that tau aggregates can also cross the cell membrane and transfer between cells (Clavaguera et al., 2009; Frost et al., 2009). These findings established the new concept that extracellular tau might be taken up by cells and induce intracellular tau accumulation and subsequent spreading of tau pathology. Therefore the mechanism of tau secretion is of potential relevance to pathogenesis of tauopathies. Nevertheless, several issues are poorly understood. First, previous studies have predominantly been performed using mice or cells overexpressing tau, and there is little evidence that endogenous tau is physiologically released into the extracellular space. Second, it is unclear whether total tau levels in brain are related to the concentration of tau in the ISF and CSF. Third, it is unknown 
whether extracellular tau levels in the ISF and CSF change together in relation to tau pathology. Fourth, no current methods have been described dynamically assess tau in living/behaving animals. Microdialysis allows in vivo sampling of molecules in the extracellular space. In this study, we have modified a microdialysis technique previously used to assess ISF A $\beta$ to assess tau from awake and freely moving mice. We validate this new methodology and provide evidence that tau is released in vivo in the absence of neurodegeneration, and that ISF tau is significantly higher than in CSF. ISF tau levels in the presence or absence of tau aggregates were also investigated using P301S tg mice. These mice showed a marked drop in ISF tau coincident with intracellular tau aggregation, whereas CSF tau increased. Together, these data suggest that monomeric ISF tau is in equilibrium with either intracellular or extracellular tau aggregates.

\section{Materials and Methods}

Recombinant proteins and antibodies. The longest mouse recombinant tau isoform mTau40 (432 aa) and the longest human tau isoform hTau40 (441 aa) were produced in the laboratory of Eva Mandelkow and used as standards in the tau ELISA. The mouse monoclonal antibody Tau-5, which recognizes both human and mouse tau (epitope at residues $218-$ 225), was from the laboratory of L. Binder (LoPresti et al., 1995; Porzig et al., 2007). Monoclonal antibodies HJ8.1 and HJ9.3 are mouse monoclonal antibodies raised by immunizing against human tau and mouse tau, respectively, in tau knock-out mice (The Jackson Laboratory). Both antibodies recognize mouse and human tau on Western blots, by immunoprecipitation, and in ELISA assays. HJ9.3 recognizes the microtubule binding region (MTBR) of tau. The epitope of HJ8.1 has not yet been defined but is outside of the MTBR. Mouse monoclonal antibody BT-2, which also recognizes human and mouse tau (epitope at residues 194198), was obtained from Pierce.

Animals. All experimental procedures involving animals were performed in accordance with guidelines established by the Animal Studies Committee at Washington University.

P301S tg mice (line PS19), which overexpress P301S human T34 isoform tau (1N4R), have been generated and characterized previously (Yoshiyama et al., 2007) and are on a B6C3 background. Age and genetic background matched nontransgenic mice littermates were used as wildtype mice. Tau knock-out mice were obtained from The Jackson Laboratory (Dawson et al., 2001). In all experiments, both male and female were used in this study.

In vitro tau microdialysis. In vitro tau microdialysis using synthetic $\mathrm{A} \beta$ solution (American Peptide) or recombinant tau solution was performed with a few modifications from our previous method (Cirrito et al., 2003). A $2 \mathrm{~mm} 100 \mathrm{kDa}$ cutoff membrane (CMA12, CMA) was connected to a microdialysis peristaltic pump with two channels (MAB20; SciPro), which was operated in a push-pull mode. As a perfusion buffer, $25 \%$ human albumin solution (Baxter) was diluted to $4 \%$ with artificial CSF (aCSF) (1.3 mм CaCl $2,1.2 \mathrm{~mm} \mathrm{MgSO}_{4}, 3 \mathrm{~mm} \mathrm{KCl}, 0.4 \mathrm{~mm} \mathrm{KH}_{2} \mathrm{PO}_{4}, 25$ $\mathrm{mm} \mathrm{NaHCO}_{3}$, and $122 \mathrm{~mm} \mathrm{NaCl}, \mathrm{pH} 7.35$ ) on the day of use and filtered through $0.1 \mu \mathrm{m}$ membrane.

In vivo tau microdialysis. In vivo microdialysis experiments to assess brain ISF tau levels from awake and freely moving mice were developed with modifications of our previously described method (Cirrito et al., 2003). A guide cannula (CMA12 guide, CMA) was stereotaxically implanted in the left hippocampus under isoflurane anesthesia, and cemented. After implantation of the cannula, mice were habituated to microdialysis cages for $2 \mathrm{~d}$ before starting ISF collection. After this recovery period, a CMA12 microdialysis probe was inserted through the guide cannula and microdialysis sample collection was started. To avoid tissue damage, the experimental window was set from 15 to $48 \mathrm{~h}$ after probe implantation. We have confirmed that within this timeframe ISF tau concentrations remain constant under constant light conditions. ISF samples were collected in a refrigerated fraction collector (Scipro) and analyzed by ELISA at the completion of each experiment. The percentage recovery in extrapolated zero flow experiments was calculated by divid- ing tau concentration at each flow rate by the extrapolated actual concentration of tau (Menacherry et al., 1992).

Preparation of tau aggregates. The MTBR of recombinant tau was prepared as described previously (Goedert and Jakes, 1990) via exploiting the heat stability of tau protein followed by cation exchange chromatography. Single-use aliquots were stored at $-80^{\circ} \mathrm{C}$ in $10 \mathrm{~mm}$ HEPES, $\mathrm{pH}$ 7.4 , and $100 \mathrm{~mm} \mathrm{NaCl}$. To induce fibrillization of tau monomer, $8 \mu \mathrm{M}$ tau MTBR was preincubated at room temperature in $2 \mathrm{~mm}$ dithiothreitol for $45 \mathrm{~min}$ followed by incubation at $37^{\circ} \mathrm{C}$ in $10 \mathrm{~mm}$ HEPES, pH 7.4, $100 \mathrm{~mm}$ $\mathrm{NaCl}$, and $8 \mu \mathrm{M}$ heparin for $18 \mathrm{~h}$ without agitation. Recombinant MTBR was then dialyzed in 10,000 molecular weight cutoff Slide-A-Lyzer Dialysis cassettes (Thermo Scientific) against aCSF. Immediately before infusion, MTBR fibrils were sonicated using the Sonicator 3000 (Misonex) at 1.5 power for $6 \times 2 \mathrm{~s}$ pulses.

Tau aggregate infusion. Infusion experiments were performed through the injection port attached to the guide cannula. PEEK tubing (Small Parts) was used for infusions and attached to CMA12 guide cannula with Elmer's Super-Fast Epoxy Resin. The end of the injection port is located at the same level as a tip of CMA12 guide cannula, which allows the infused solution to exit surrounding the CMA12 probe. Before use, the infusion line was washed with aCSF and coated by running $0.15 \%$ BSA in aCSF to prevent nonspecific binding of proteins to the inner wall of the tubing. After collecting baseline tau for $7.5 \mathrm{~h}$ at the flow rate of $1.0 \mu \mathrm{l} / \mathrm{min}$ from 3.5- to 6-month-old P301S tg mice, a total volume of $2 \mu$ l of MTBR fibrils ( $8 \mu \mathrm{M}$ monomer equivalent) per infusion was delivered into the hippocampus at $0.07 \mu \mathrm{l} / \mathrm{min}$ by Hamilton syringe connected to a microdialysis infusion syringe pump (Stoelting). The same volume of aCSF was delivered as a vehicle control. Microdialysis fluid was collected for $1.5 \mathrm{~h}$ after the injection was completed, and tau levels were assessed by ELISA. The presence of MTBR tau aggregates surrounding the probes at the termination of the experiments was confirmed by performing immunostaining with anti-HA antibody.

Tau ELISA. The tau concentration in microdialysis samples were analyzed in a tau sandwich ELISA. Two micrograms of Tau-5 antibody directed against amino acids $218-225$ of tau were coated per well in a 96-well plate (Nunc) and incubated overnight at $4^{\circ} \mathrm{C}$. The plate was blocked with $4 \% \mathrm{BSA}$ for $60 \mathrm{~min}$ at $37^{\circ} \mathrm{C}$. Microdialysis samples and brain extracts and standards diluted with our standard buffer $(0.25 \%$ BSA, $300 \mathrm{~mm}$ Tris, $0.05 \%$ azide, supplemented with protease inhibitors in PBS) were loaded and incubated overnight at $4^{\circ} \mathrm{C}$. To assess tau concentration in ISF, the longest mouse tau isoform mTau40 (432 aa) was used as a standard. Seventy percent formic acid (FA) extracts were neutralized initially by 1:20 dilution into $1 \mathrm{~m}$ Tris phosphate buffer, $\mathrm{pH} 11$, and then diluted by standard buffer as described previously (Kawarabayashi et al., 2001). On the next day, biotinylated BT-2 antibody (Pierce) was applied onto the plate as a detection antibody at a concentration of $0.3 \mu \mathrm{g} / \mathrm{ml}$ and incubated for $90 \mathrm{~min}$ at $37^{\circ} \mathrm{C}$, followed by incubation with streptavidinpoly-horseradish peroxidase- 40 (Fitzgerald) for $90 \mathrm{~min}$ at room temperature. All assays were developed using Super Slow ELISA TMB (Sigma) and read on a Bio-Tek FL-600 plate reader at $650 \mathrm{~nm}$. Recombinant mouse tau was used as a standard. For CSF samples, a 96-well plate with half area per normal-sized well was used to minimize the volume loaded per well.

Brain extraction and CSF collection. Mice were perfused with chilled PBS-heparin, and CSF was collected from the cisterna magna as previously described (DeMattos et al., 2002). The left hemisphere was collected for immunostaining and fixed overnight in $4 \%$ paraformaldehyde at $4^{\circ} \mathrm{C}$ and then transferred to $30 \%$ sucrose. The right hemisphere was dissected to isolate the hippocampus for biochemical analysis. Hippocampus was kept at $-80^{\circ} \mathrm{C}$ until analyzed. Hippocampus was weight and homogenized in RAB buffer [ $100 \mathrm{~mm}$ MES, $1 \mathrm{~mm}$ EDTA, $0.5 \mathrm{~mm}$ $\mathrm{MgSO}_{4}, 750 \mathrm{~mm} \mathrm{NaCl}, 20 \mathrm{~mm} \mathrm{NaF}, 1 \mathrm{mM} \mathrm{Na}_{3} \mathrm{VO}_{4}$, supplemented by protease inhibitor (Complete, Roche) and phosphatase inhibitor (PhosSTOP,Roche)]. The samples were centrifuged at 50,000 $\times g$ for 20 min at $4^{\circ} \mathrm{C}$. The supernatants were collected as RAB soluble fractions. The pellets were resuspended in RIPA [ $150 \mathrm{~mm} \mathrm{NaCl}, 50 \mathrm{~mm}$ Tris, $0.5 \%$ deoxycholic acid, 1\% Triton X-100, 0.5\% SDS-25 mm EDTA, pH 8.0, supplemented by protease inhibitor (Complete, Roche) and phosphatase inhibitor (PhosSTOP, Roche)] and centrifuged at 50,000 $\times g$ for $20 \mathrm{~min}$ 
at $4^{\circ} \mathrm{C}$. The supernatants were collected as RIPA soluble fractions. The pellets were resuspended in $70 \%$ formic acid and centrifuged at 50,000 $\times$ $g$ for $20 \mathrm{~min}$ at $4^{\circ} \mathrm{C}$. The supernatants were collected as $70 \%$ formic acid fractions. All fractions were used for immunoblot analysis.

Immunoblot analysis. Fractionated brain extracts were dissolved in a sample buffer containing $2 \% \beta$-mercaptoethanol and $2 \%$ SDS. Before applying sample buffer, $70 \%$ formic acid fractions were neutralized by diluting $1: 3$ with $1: 1$ mixture of $10 \mathrm{~N} \mathrm{NaOH}$ and neutralization buffer ( 1 $\mathrm{mol} / \mathrm{L}$ Tris base; $0.5 \mathrm{~mol} / \mathrm{L} \mathrm{NaH}_{4} \mathrm{PO}_{4}$ ) as described previously (Clinton et al., 2010). Proteins were separated by reducing $4-12 \%$ Bis-Tris mini-gel (Invitrogen), transferred to PVDF membrane, and incubated with BT-2 antibody (Pierce) or anti-actin (Sigma), followed by HRP-conjugated anti mouse antibody (GE Healthcare Pharmacia Biotech). Bands were visualized with Lumigen-TMA6 (GE Healthcare).

Immunoprecipitation and immunoblot analysis. Hippocampal microdialysis samples were collected at $1.0 \mu \mathrm{l} / \mathrm{min}$ for $15 \mathrm{~h}$ from P301S tau transgenic mouse and wild-type mice. ISF was immunoprecipitated by Dynabeads (Invitrogen) coated with HJ8.1 or HJ9.3 tau antibody according to the manufacturer's instructions. Precipitated fractions were loaded on a reducing $4-12 \%$ Bis-Tris mini-gel (Invitrogen) and transferred to nitrocellulose membrane. Biotinylated BT-2 antibody (Pierce) and PolyHRP-conjugated streptavidin (Thermo Scientific) were used to eliminate the interference of precipitated antibodies.

Statistical analysis. Data in figures represent mean \pm SEM. All statistical analysis was performed using Prism version 5.04 for Windows (GraphPad). Differences in ISF tau levels were analyzed using a one-way ANOVA followed by post hoc test.

\section{Results}

Development and validation of a tau microdialysis technique in wild-type and P301S tau transgenic mice

To determine whether we could measure recombinant tau via microdialysis in vitro, probes with $100 \mathrm{kDa}$ cutoff membranes were used. Higher molecular weight cutoff membrane sizes usually cause fluid loss from the probe. Generally, this phenomenon is caused by hydrostatic pressure created inside of the probe (Trickler and Miller, 2003). To minimize the fluid loss from microdialysis probes, we increased the osmotic pressure of the perfusion buffer by using a $4 \%$ albumin solution. We also operated a peristaltic pump in a push-pull mode to prevent pressure from being created. These technical changes from our prior method to assess $\mathrm{A} \beta$ (Cirrito et al., 2003) were first tested by performing in vitro $\mathrm{A} \beta$ microdialysis using $100 \mathrm{kDa}$ cutoff probes. We found that $4 \%$ albumin increased the measured $\mathrm{A} \beta$ concentration in the dialysate by fourfold compared to $0.15 \%$ BSA, which we normally use for $A \beta$ microdialysis (data not shown). The use of a push-pull pump also improved the recovery by 1.6-fold compared to a push pump. These technical improvements also allowed us to collect recombinant tau through $100 \mathrm{kDa}$ cutoff microdialysis probes in vitro (data not shown). The ISF tau being measured is soluble monomeric tau due to the cutoff size of the microdialysis membrane.

We next evaluated the microdialysis technique in vivo. We first addressed whether tau is normally present in the brain ISF in the absence of neurodegeneration. To address this issue, in vivo tau microdialysis was initiated in both wild-type mice and in 3-month-old P301S tg mice, at an age before the appearance of insoluble tau and neurodegeneration. In this study, we focused our investigations on hippocampus, which is one of the regions affected by tau pathology relatively early during aging (Braak and Braak, 1997; Duyckaerts and Hauw, 1997) as well as in different tauopathies. The mice were implanted with microdialysis probes and ISF was sampled. The tau concentration in the collected dialysate was quantified by Tau-5/BT-2 ELISA. This ELISA recog-
A
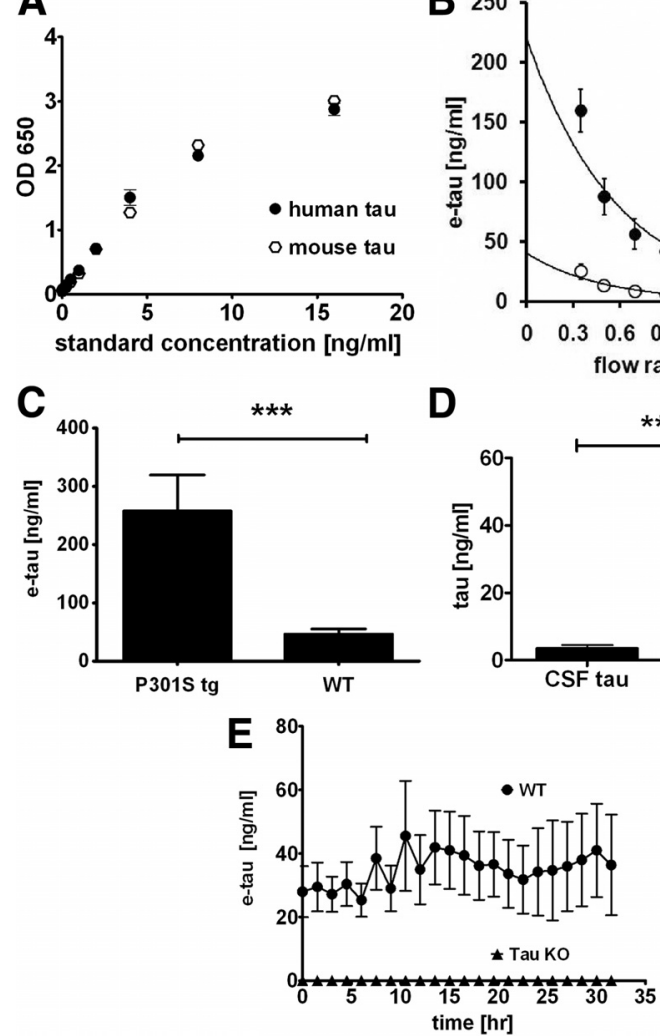

Figure 1. e-tau in 3-month-old wild-type mice and P301S tau tg mice. A, Tau-5/BT-2 ELISA recognizes recombinant human tau and mouse tau equivalently. $n=4$. $B$, At varying flow rates of the microdialysis perfusion buffer from $0.35 \mu \mathrm{l} / \mathrm{min}$ to $1.5 \mu \mathrm{l} / \mathrm{min}$, e-tau was quantified by ELISA in the hippocampus in wild-type (WT, $n=12$ ) and P301S $\operatorname{tg}$ (P301S tg, $n=7$ ) mice. C, The mean e-tau levels were calculated by the zero flow method. ${ }^{* * *} p<0.0001$ by unpaired $t$ test. $\boldsymbol{D}$, ISF $(n=13)$ and CSF $(n=8)$ tau levels were measured in 3-month-old wild-type mice. ${ }^{* *} p<0.01$ by unpaired $t$ test. $\boldsymbol{E}$, e-tau levels was determined in 3-month-old wild-type mice (WT, $n=5$ ) and tau knock-out mice (Tau K0, $n=5$ ).

nizes mouse tau and human tau equivalently, which allows us to quantify total tau levels in ISF (Fig. $1 A$ ).

Interestingly, we were able to detect monomeric tau in ISF from both P301S tg mice and wild-type littermates measured as frequently as every hour. We determined the in vivo absolute concentration of monomeric ISF tau by the extrapolated zero flow method (Cirrito et al., 2003; Kim et al., 2009). In this method, we varied the flow rate of microdialysis perfusion buffer (Fig. $1 B$ ). The relative recovery of tau and other molecules is inversely related to the flow rate. Therefore the concentration of tau recovered by microdialysis, i.e., exchangeable tau (e-tau), can be determined by extrapolating the recovery curve back to theoretical zero flow rate, which represents the estimated in vivo concentration of ISF tau. Utilizing this method, the monomeric ISF e-tau concentration is $44.9 \pm 9.4 \mathrm{ng} / \mathrm{ml}$ in 3-month-old wildtype mice and $248.8 \pm 54.2 \mathrm{ng} / \mathrm{ml}$ in P301S tg mice (Fig. $1 \mathrm{~B}, \mathrm{C}$ ). This fivefold difference is consistent with the expression level of human tau being approximately fivefold higher in P301S tg mice than in endogenous murine tau (Yoshiyama et al., 2007). The concentrations of e-tau in the ISF of wild-type mice are $\sim 10$-fold higher than the levels found in mouse CSF (Fig. 1D). Given that both wild-type and P301S mice show no evidence of neurodegeneration at this age, and that we have previously shown that there is no significant cell death caused by microdialysis probe insertion (Cirrito et al., 2003), these data suggest that tau is normally 


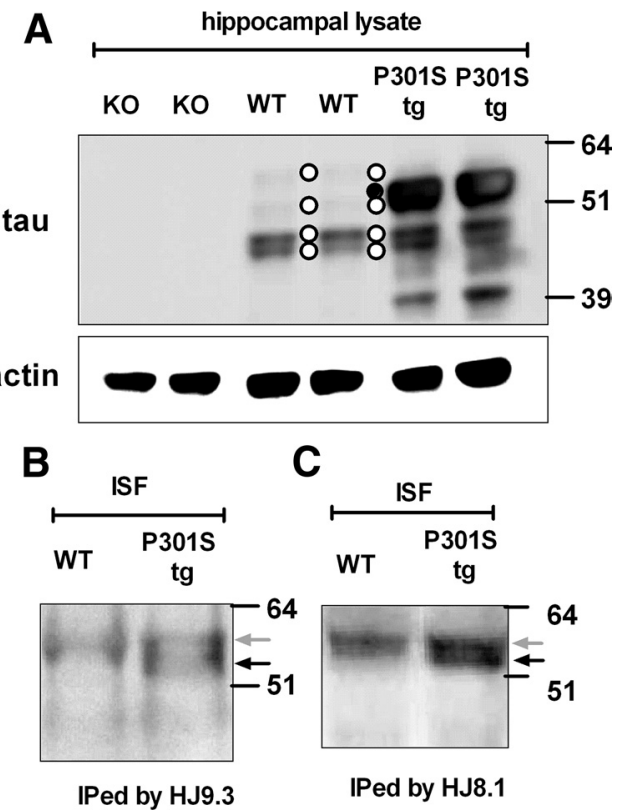

Figure 2. Full-length tau is present in ISF of wild-type and P301S tg mice. A, Hippocampal lysates from Tau KO (KO), wild-type (WT), and P301S tg (P301S tg) mice were analyzed by immunoblot with the anti-tau antibody BT-2 or anti-actin antibody. Thirteen micrograms of protein were loaded per well. Four bands corresponding to endogenous murine tau and one band corresponding to human tau are indicated as white circles and a black circle, respectively. There is also a $39 \mathrm{kDa}$ band representing a form of human tau in the P3015 tg hippocampal lysate. This may represent a tau degradation product. ISF tau from wild-type (WT) and P301S tg (P3015 tg) mice was immunoprecipitated by anti-tau monoclonal antibodies HJ9.3 (B) or HJ8.1 (C) and analyzed by immunoblot. The bands were visualized by biotinylated BT-2 antibody. The gray and black arrows indicate endogenous murine tau and human tau, respectively.

released from cells into the extracellular space of brain under physiological conditions. Further, the specificity of our assay was confirmed by performing microdialysis on tau knock-out mice. There is no detectable tau in these mice at any time examined (Fig. 1E).

\section{Full-length tau is present in ISF}

To determine the major tau species in ISF recovered by the microdialysis probe, we immunoprecipitated tau from the ISF samples of both wild-type mice and P301S tg mice using tau antibodies recognizing both mouse and human tau. We chose to use two anti-tau monoclonal antibodies that worked well in immunoprecipitation assays as the amount of monomeric tau in ISF is still relatively low. Following immunoprecipitation, we analyzed tau by immunoblot. Endogenous murine tau isoforms migrate at 48-62 kDa (Takuma et al., 2003). In wild-type brain lysate, tau appeared in four separate bands on SDS-PAGE (Fig. $2 A)$. The most abundant species in wild-type mice migrated at $\sim 48 \mathrm{kDa}$. In P301S tg mice brain, in addition to the four endogenous murine tau bands, overexpressed human 1N4R tau was observed as an intense band migrating at $\sim 55 \mathrm{kDa}$ as well as a 39 $\mathrm{kDa}$ band, which may represent a tau degradation product.

In contrast to total brain lysates, upon immunoprecipitation, a single tau band was detected with antibody HJ9.3 recognizing the MTBR region of tau in ISF from wild-type mice (Fig. $2 B$ ). This band corresponded to the largest isoform $2 \mathrm{~N} 4 \mathrm{R}$ observed in mouse brain lysate. In ISF of P301S tg mice, a human-specific tau band was coprecipitated with the aforementioned mouse tau band and was slightly lower in molecular weight (Fig. $2 B$ ). These two bands were also precipitated by another mouse monoclonal
A
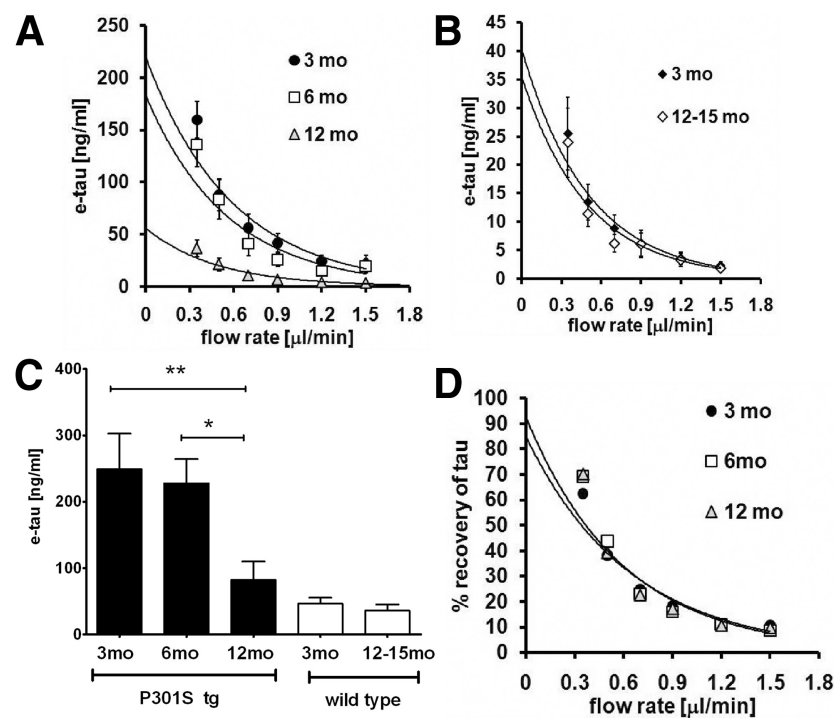

Figure 3. e-tau in P301S tg mice and wild-type mice at different ages. $\boldsymbol{A}$, Zero flow experiments were performed in $3-(3 \mathrm{mo}, n=12), 6-(6 \mathrm{mo}, n=12)$, and $12-(12 \mathrm{mo}, n=8)$ month-old P301S tg mice. Tau levels at each flow rate were quantified by ELISA. $\boldsymbol{B}$, Zero flow experiments were performed in 3- $(3 \mathrm{mo}, n=12)$ and 12 - to 15 - $(12-15 \mathrm{mo}, n=6)$ month-old wild-type mice. Tau levels at each flow rate were quantified by ELISA. $C$, The mean e-tau was assessed in 3- $(3 \mathrm{mo}, n=12), 6-(6 \mathrm{mo}, n=8)$, and $12-(12 \mathrm{mo}, n=8)$ month-old P3015 tg mice and 3 - $(3 \mathrm{mo}, n=12)$ and 12 - to $15-(12-15 \mathrm{mo}, n=6)$ month-old wild-type mice by the zero flow method. ${ }^{*} p<0.05,{ }^{* *} p<0.01$. D, The percentage recovery of tau from P301S tg mice was calculated and plotted against flow rates. There is no difference in the percentage recovery between groups.

antibody raised against tau HJ8.1 (Fig. 2C). These data suggested that the major species in ISF that we are assessing by ELISA is likely full-length monomeric tau.

\section{Monomeric ISF Tau decreases in P301S Tau transgenic but not wild-type mice over time}

We hypothesized that monomeric ISF tau levels would be influenced by the presence of tau aggregates. To test this hypothesis, we assessed monomeric ISF tau in both P301S tg and wild-type mice at different ages. P301S tg mice begin to accumulate filamentous tau at $\sim 6$ months of age, which then progressively accumulates in an age-dependent manner (Yoshiyama et al., 2007).

The in vivo concentration of monomeric ISF tau in 3-, 6-, and 12-month-old P301S tg mice was determined by the zero flow method (Fig. 3A). ISF tau decreased in an age-dependent manner (Fig. $3 A, C$ ). P301S tg mice at both 3 and 6 months have tau concentrations of $248.8 \pm 54.2$ and $227.4 \pm 36.8 \mathrm{ng} / \mathrm{ml}$, respectively. In contrast, monomeric ISF tau levels dropped by $\sim 70 \%$ to $72.3 \pm 28.0 \mathrm{ng} / \mathrm{ml}$ at 12 months of age. Monomeric ISF tau levels were also measured in 3 and 12- to 15-month-old wild-type mice (Fig. $3 B$ ). No age-dependent change of monomeric ISF tau levels in wild-type mice was detected (Fig. $3 B, C$ ). We also compared the percentage recovery of monomeric ISF tau at each flow rate at all ages of P301S tg mice. There was no change in the percentage recovery, ruling this out as an explanation for the drop in monomeric ISF tau in the P301S tg mice (Fig. 3D). Together, these data suggest that the dramatic drop in monomeric ISF tau levels with age in P301S tg mice is linked to tau aggregate formation and accumulation.

\section{CSF tau in P301S human tau transgenic mice}

Tau is present in human CSF (Vandermeeren et al., 1993; Perrin et al., 2009). Thus, we determined the tau concentrations in CSF 

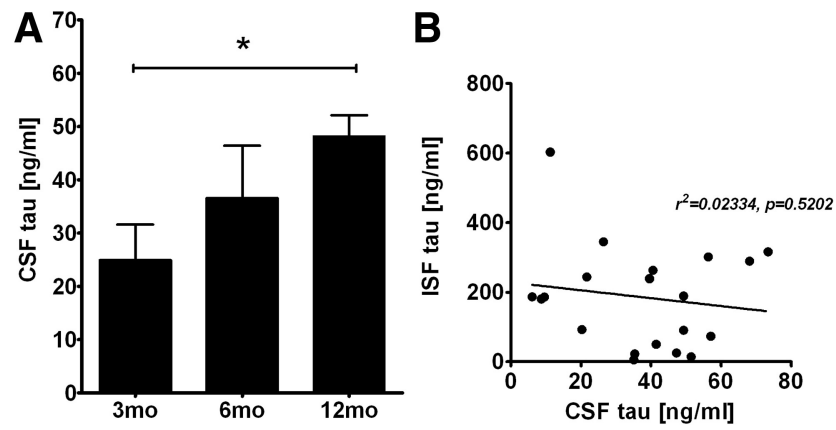

Figure 4. CSF tau concentration in P301S tg mice. $A$, CSF tau levels in 3- (3mo, $n=10)$, 6 $(6 \mathrm{mo}, n=5)$, and $12-(12 \mathrm{mo}, n=8)$ month-old P301S tg mice were quantified by ELISA. ${ }^{*} p<$ 0.05. B, Relationship between ISF tau and CSF tau in P301S tg mice. There is no significant correlation between ISF tau and CSF tau.

of P301S tg mice. In contrast to ISF, we found that CSF tau in $\mathrm{P} 301 \mathrm{~S}$ tg mice increased in an age-dependent manner (Fig. $4 \mathrm{~A}$ ). CSF tau was $24.9 \pm 6.4$ and $36.5 \pm 12.4 \mathrm{ng} / \mathrm{ml}$ in 3 - and $6-\mathrm{month}-$ old P301S tg mice, respectively, whereas it was $48.2 \pm 3.7 \mathrm{ng} / \mathrm{ml}$ in 12-month-old P301S tg mice. In 3-month-old P301S tg mice, CSF tau was $\sim 10 \%$ of tau measured in ISF.

Since there is no diffusion barrier between ISF and CSF, we wondered whether the increase in CSF tau correlated with changes in ISF tau levels. We assessed hippocampal ISF and CSF tau in individual mice. If these two pools were directly related to each other, a negative correlation would be expected. However there was no correlation between ISF and CSF tau, suggesting independent regulation (Fig. $4 B$ ).

\section{Age-dependent decrease of total brain soluble tau in P301S tau transgenic mice} P301S tg mice develop tau pathology in an age-dependent manner (Yoshiyama et al., 2007). The decrease of monomeric ISF tau, along with the presence of tau pathology, led us to hypothesize that tau aggregates decrease soluble tau. To determine whether soluble tau is decreased with age in P301S tg mice, hippocampal tissue was sequentially extracted with RAB and RIPA buffer, followed by $70 \%$ FA solubilization, and analyzed by immunoblot.

In wild-type mice, endogenous murine tau was collected exclusively in the first two fractions RAB and RIPA, and there was no detectable tau in the 70\% FA soluble fraction (Fig. 5A). Human and mouse tau was also observed in the RAB and RIPA fractions in 3- and 6-month-old P301S tg mice with no tau present in the insoluble fraction by immunoblot. In contrast, in 12month-old P301S tg mice, there was a decrease of human tau in the RAB and RIPA fractions. (Fig. 5A).

To assess tau levels in a more quantitative fashion, tau levels in each fraction were also measured by ELISA (Fig. 5B-D). Consistent with immunoblot analysis, tau levels in the RAB fraction were decreased by $32 \%$ in 6-month-old mice and by $52 \%$ in 12-month-old mice compared to 3-month-old P301S tg mice. Tau levels in the RIPA fraction were also decreased by $38 \%$ in 12-month-old P301S tg mice compared to 3-month-old mice. In contrast, insoluble tau in $70 \%$ formic acid fractions was increased by 3.5-fold in 12-month-old P301S tg mice. These data suggest that the insoluble brain tau results in a decrease in brain soluble tau, which corresponds to a reduction of ISF tau.

\section{Tau aggregates decrease monomeric ISF tau in vivo}

A previous study with cultured cells suggested that tau aggregates are released into the extracellular space (Frost et al., 2009). Thus
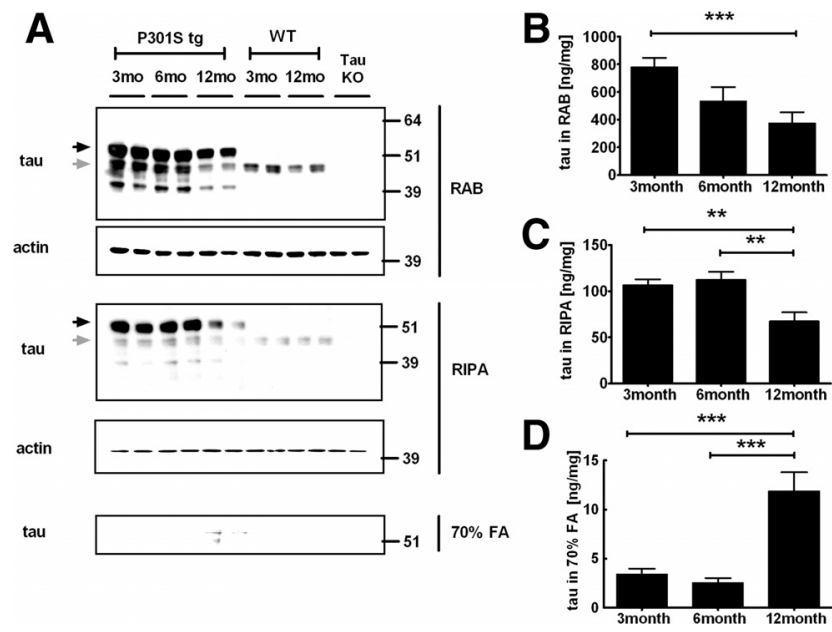

Figure 5. Hippocampal soluble and insoluble tau levels in P301S tg mice. $\boldsymbol{A}$, The hippocampus from P301S tg (P301S tg), wild-type (WT), and Tau KO (Tau KO) mice were sequentially extracted by RAB, RIPA, 70\% FA, and extracted proteins were analyzed by immunoblot with BT-2 (anti-tau) or anti-actin antibody as a control. Black and gray arrows indicate human tau and endogenous murine tau, respectively. Tau levels in RAB fractions $(\boldsymbol{B}), \operatorname{RIPA}$ fractions $(\boldsymbol{C})$, and $70 \%$ formic acid fractions $(\boldsymbol{D})$ were quantified by ELISA. $n=5-8,{ }^{* *} p<0.01,{ }^{* * *} p<0.001$.
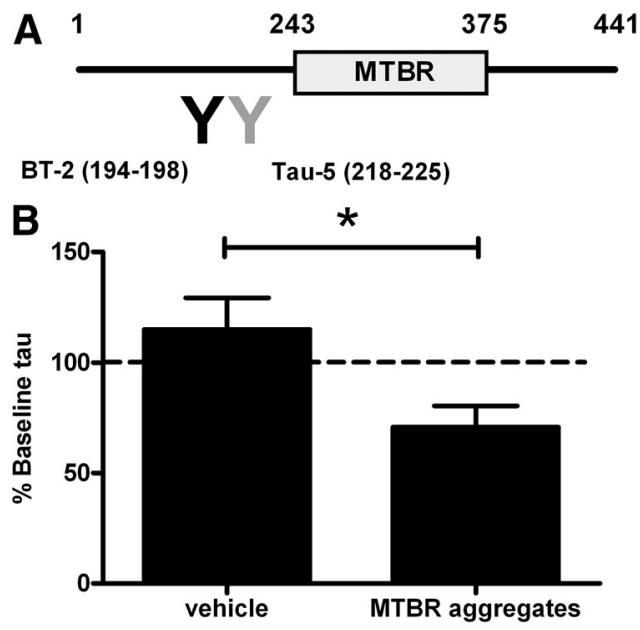

Figure 6. Tau aggregates induce an acute decrease of ISF tau levels in 3-month-old P301S tg mice. $\boldsymbol{A}$, Tau aggregates containing the MTBR. MTBR is not recognized by tau antibodies (BT-2, Tau-5) used in ELISA. $\boldsymbol{B}$, Relative e-tau levels after injection of vehicle $(n=3)$ or MTBR aggregates $(n=4)$ in P301S tg mice were measured by ELISA. * $p<0.05$.

we hypothesized that extracellular tau aggregates, if present, might decrease monomeric ISF tau. We prepared tau aggregates in vitro and injected them into the brain while simultaneously measuring soluble monomeric ISF tau levels. We used a form of truncated tau that comprises the repeat domain of the MTBR. The repeat domain of tau readily forms filaments because it lacks the $\mathrm{N}$ - and C-terminal flanking domains and exposes the core with high $\beta$-propensity (von Bergen et al., 2000), and thus MTBR can seed fibril formation of tau monomer in vitro (Frost et al., 2009). Because MTBR tau aggregates lack the epitopes recognized by the BT-2 and Tau-5 antibodies used in our tau ELISA, this injection strategy allowed specific detection of endogenous monomeric ISF tau (Fig. 6A).

After collecting baseline monomeric ISF tau, MTBR tau aggregates or vehicle were infused around the microdialysis probe through an injection port attached to microdialysis guide cannula. The monomeric ISF tau concentration before and after in- 
A

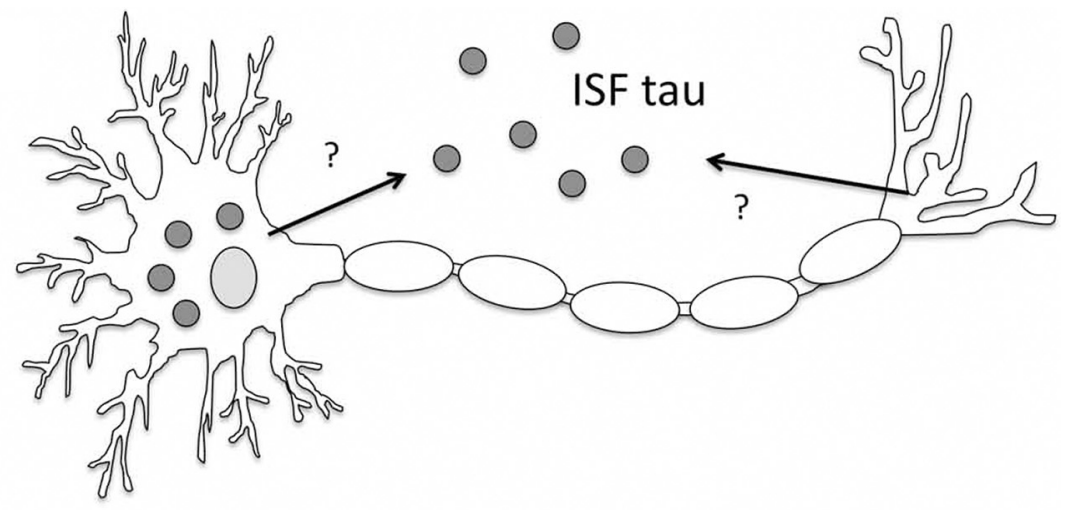

B

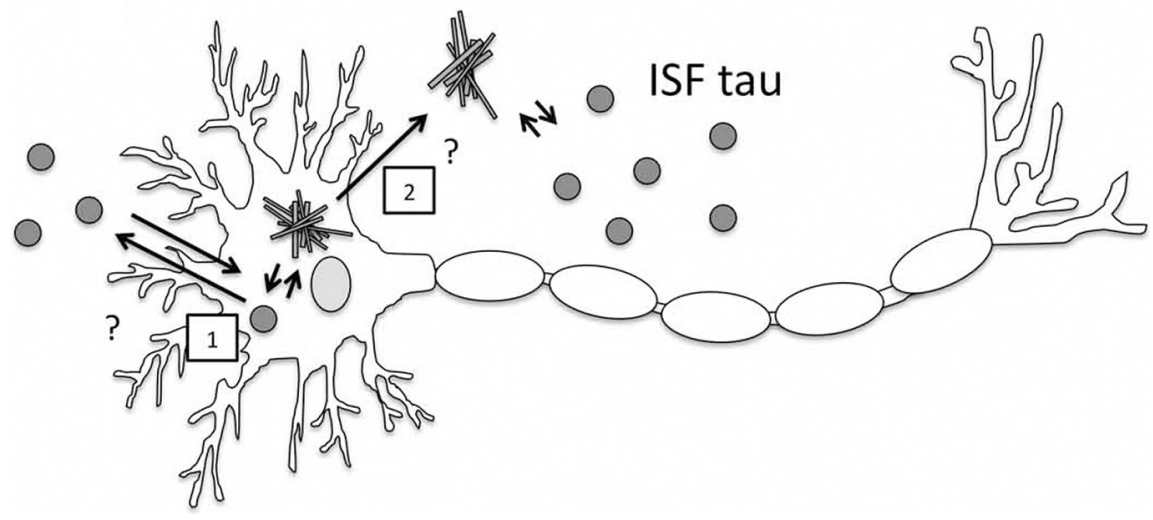

Figure 7. Proposed diagram of tau release in the presence or absence of tau aggregates. $\boldsymbol{A}$, Tau is released from neurons under normal conditions. $\boldsymbol{B}$, Once tau aggregates form inside (1) or potentially outside (2) of cells, they can sequester intracellular and extracellular soluble tau and lower the level of monomeric tau in the extracellular space.

jection was measured by ELISA. The injection of tau aggregates reduced ISF tau levels by $30 \%$ compared to baseline monomeric ISF tau levels (Fig. $6 \mathrm{~B}$ ). In contrast, vehicle infusion did not alter monomeric ISF tau levels. When infused in ISF of wildtype mice, MTBR aggregates did not change endogenous murine tau levels, suggesting that $\mathrm{P} 301 \mathrm{~S}$ tau with higher aggregation potential is preferentially sequestered by aggregates (data not shown). Together, these data suggested that extracellular tau aggregates, if present, can decrease soluble monomeric ISF tau.

\section{Discussion}

We have demonstrated that monomeric tau is normally present in the brain ISF and can be measured on an hourly basis using microdialysis. Its levels in ISF are much higher than in CSF and levels in ISF parallel the levels of tau in brain homogenates. Utilizing P301S tg mice, we found that monomeric ISF tau is dramatically decreased following the onset of tau aggregation. Supporting the idea that the decrease in ISF tau is due to an equilibrium between either intracellular or extracellular tau aggregates, infusions of aggregated tau result in a rapid decrease in monomeric ISF tau.

We provide direct evidence that monomeric tau is present in ISF of wild-type mice, even in the absence of neurodegeneration. Even though tau lacks a signal sequence, its release into the extracellular space appears to be a normal process. These data are consistent with the previous observation that tau is present in CSF of humans without neurodegeneration.

The level of monomeric tau present in the ISF was paralleled the level of tau protein in the brain. Tau protein is approximately fivefold higher in P301S tg mice brain than in wild-type littermates, and similar differences between P301S tg mice and wild-type mice were found in ISF. This is important because it suggests that the levels of intracellular and extracellular tau are related. We explored the form of tau present in ISF by immunoprecipitation. It was observed that tau species, which appear to correspond to full-length tau, were present in ISF of both wild-type mice and P301S tg mice. Tau was immunoprecipitated with two different antibodies recognizing different regions of tau and migrated at molecular weights consistent with them being full-length tau. Most regions of tau also have been identified in human CSF using mass spectrometry as well as the media of cultured cells (Portelius et al., 2008; Kim et al., 2010a). Immunoprecipitated ISF tau from wild-type mice was higher in molecular weight than the most abundant tau isoform present in brain lysates. This suggests that there might be a particular isoform or posttranslationally modified form of tau that is released from cells in a regulated fashion. Recent work also indicated that release of tau depends on the N-terminal half of tau (Kim et al., 2010b).

The mechanism whereby tau is released into the extracellular space is still unknown. Besides tau, there are increasing numbers of intracellular proteins that lack ER signal sequences but reach the extracellular spaces via an unconventional secretion pathway (Nickel, 2010). Even though tau lacks a transmembrane or obvious secretion signal sequence, some tau has been observed in membranous compartments such as the Golgi body, plasma membranes, and mitochondria, which could be linked to secretion (Jung et al., 1993; Brandt et al., 1995; Farah et al., 2006). Exosome-mediated secretion, which has been suggested as a mechanism of $\alpha$-synuclein secretion, could also play a role (Emmanouilidou et al., 2010). While it is certainly possible that tau dimers, trimers, oligomers, or even tau exosomes exist in ISF, our current method would not allow for the direct assessment of these structure, since the dialysis probe cutoff is $100 \mathrm{kDa}$. Recent findings provide strong evidence that soluble tau, possibly an oligomer, can cause neuronal dysfunction independent of NFTs (Oddo et al., 2006; Thies and Mandelkow, 2007; de Calignon et al., 2010; Hoover et al., 2010). For example, synaptic failure in the P301S tg mice occurs before tangle formation (Yoshiyama et al., 2007). In other studies, cognitive dysfunction of these mice was improved by suppressing tau expression despite ongoing accumulation of NFTs, suggesting that some form of soluble tau may be critical in neurodegeneration (Santacruz et al., 2005; Mocanu et al., 2008). Other studies suggest that soluble, nonaggregated tau is a common mediator of neuronal injury due to both excitotoxicity and APP/A $\beta$ (Roberson et al., 2007, 2011; Ittner et al., 2010). This has led to the idea that suppression of tau levels as therapy. Tau microdialysis provides a dynamic in vivo method to assess pharmacodynamic effects of treatments on tau lowering. While extracellular tau may generally reflect levels of intracellular 
tau, the potential role of extracellular tau in any of the toxic or other properties of tau, if any, is unknown. As some studies have reported a cytotoxic effect of extracellular tau when it is added to culture medium (Gómez-Ramos et al., 2006, 2008), it will be important to examine the effect of soluble extracellular tau on neuronal dysfunction.

Intriguingly, recent studies have shown that propagation of tau aggregation from the outside to the inside of the cell occurs in vivo and in cell culture models. This could explain the vulnerability of specific networks in tauopathies and other neurodegenerative diseases (Frost and Diamond, 2010). It is noteworthy that anti-tau immunotherapy has beneficial effects in mouse models of tauopathy (Asuni et al., 2007; Sigurdsson, 2009; Boutajangout et al., 2010). While the mechanism underlying tau immunotherapy has not yet been clarified, if extracellular tau is the target molecule, tau microdialysis may provide an opportunity to better assess therapeutic mechanisms.

We found that ISF tau levels dramatically decrease from 6 to 12 months of age in P301S tg mice. This change with age was not seen in the wild-type mice, suggesting that it is disease related. It seems most likely that ISF tau is in equilibrium with extracellular, intracellular tau aggregates, or both. Indeed, we observed that soluble tau from brain homogenates of P301S tg mice was decreased in parallel with the deposition of insoluble tau. We favor a sequestration model. This was reported in another mouse model (Deters et al., 2008). Further, using regulatable tau transgenic mouse models, a dynamic equilibrium between soluble tau and insoluble tau was observed (Sydow and Mandelkow, 2010; Sydow et al., 2011). Increased clearance could also account for the decrease in ISF tau, which we will test in future studies.

We observed that exogenously administrated tau aggregates quickly reduce ISF tau levels. Tau aggregates in the extracellular space could lead to decrease ISF tau even in relatively young P301S mice. In contrast to ISF tau, CSF tau increased with age in P301S tg mice. One possibility is that CSF tau may increase with neurodegeneration. We have measured ISF tau in the hippocampus of these aged mice, where many aggregates form and where a similar reduction in ISF tau was observed. Thus, it is possible that hippocampal aggregates influence ISF tau locally, whereas CSF tau levels reflect mean changes in total extracellular tau in the CNS. Further, oligomers are not measurable in ISF with this microdialysis membrane and could result in an apparent decrease in older P301S tg mice. This is reminiscent of our previous finding that $\mathrm{A} \beta$ in hippocampal ISF of APP transgenic mice is also not correlated with $A \beta$ in CSF (Cirrito et al., 2003). Further, ISF tau is significantly higher than CSF levels. Because tau is a large protein that may be cleared prominently by cellular uptake, local degradation, and perivascular drainage (Weller et al., 2008), most of it may never reach the CSF via bulk flow or diffusion.

Herein, we propose a model to explain the dynamics of ISF tau (Fig. 7). In the absence of tau aggregates, tau is released into ISF via undefined mechanisms. In contrast, in the presence of tau aggregates, tau is sequestered within the cells, which leads to the decrease in ISF tau. Once tau aggregates are released from cells, they can also be sequestered extracellularly, decreasing ISF tau levels.

In summary, our microdialysis method enabled the measurement of extracellular tau from both wild-type and human tau transgenic mice. These data provide a link between tau metabolism, ISF tau, and tau aggregation, and this approach provides an in vivo tool to assess tau-mediated pathogenesis and treatments that influence tau levels dynamically in vivo. This method may also provide an important way to investigate the role of extracel- lular tau on the spreading of tau pathology and neuronal dysfunction.

\section{References}

Arriagada PV, Growdon JH, Hedley-Whyte ET, Hyman BT (1992) Neurofibrillary tangles but not senile plaques parallel duration and severity of Alzheimer's disease. Neurology 42:631-639.

Asuni AA, Boutajangout A, Quartermain D, Sigurdsson EM (2007) Immunotherapy targeting pathological tau conformers in a tangle mouse model reduces brain pathology with associated functional improvements. J Neurosci 27:9115-9129.

Ballatore C, Lee VM, Trojanowski JQ (2007) Tau-mediated neurodegeneration in Alzheimer's disease and related disorders. Nat Rev Neurosci 8:663-672.

Bancher C, Braak H, Fischer P, Jellinger KA (1993) Neuropathological staging of Alzheimer lesions and intellectual status in Alzheimer's and Parkinson's disease patients. Neurosci Lett 162:179-182.

Boutajangout A, Quartermain D, Sigurdsson EM (2010) Immunotherapy targeting pathological tau prevents cognitive decline in a new tangle mouse model. J Neurosci 30:16559-16566.

Braak H, BraakE (1997) Diagnostic criteria for neuropathologic assessment of Alzheimer's disease. Neurobiol Aging 18:S85-88.

Brandt R, Léger J, Lee G (1995) Interaction of tau with the neural plasma membrane mediated by tau's amino-terminal projection domain. J Cell Biol 131:1327-1340.

Cirrito JR, May PC, O’Dell MA, Taylor JW, Parsadanian M, Cramer JW, Audia JE, Nissen JS, Bales KR, Paul SM, DeMattos RB, Holtzman DM (2003) In vivo assessment of brain interstitial fluid with microdialysis reveals plaque-associated changes in amyloid- $\beta$ metabolism and half-life. J Neurosci 23:8844-8853.

Clavaguera F, Bolmont T, Crowther RA, Abramowski D, Frank S, Probst A, Fraser G, Stalder AK, Beibel M, Staufenbiel M, Jucker M, Goedert M, Tolnay M (2009) Transmission and spreading of tauopathy in transgenic mouse brain. Nat Cell Biol 11:909-913.

Clinton LK, Blurton-Jones M, Myczek K, Trojanowski JQ, LaFerla FM (2010) Synergistic interactions between $\mathrm{A} \beta$, tau, and $\alpha$-synuclein: acceleration of neuropathology and cognitive decline. J Neurosci 30:7281-7289.

Dawson HN, Ferreira A, Eyster MV, Ghoshal N, Binder LI, Vitek MP (2001) Inhibition of neuronal maturation in primary hippocampal neurons from tau deficient mice. J Cell Sci 114:1179-1187.

de Calignon A, Fox LM, Pitstick R, Carlson GA, Bacskai BJ, Spires-Jones TL, Hyman BT (2010) Caspase activation precedes and leads to tangles. Nature 464:1201-1204.

DeMattos RB, Bales KR, Parsadanian M, O'Dell MA, Foss EM, Paul SM, Holtzman DM (2002) Plaque-associated disruption of CSF and plasma amyloid-beta (Abeta) equilibrium in a mouse model of Alzheimer's disease. J Neurochem 81:229-236.

Deters N, Ittner LM, Götz J (2008) Divergent phosphorylation pattern of tau in P301L tau transgenic mice. Eur J Neurosci 28:137-147.

Duyckaerts C, Hauw JJ (1997) Prevalence, incidence and duration of Braak's stages in the general population: can we know? Neurobiol Aging 18:362-369; discussion 389-392.

Emmanouilidou E, Melachroinou K, Roumeliotis T, Garbis SD, Ntzouni M, Margaritis LH, Stefanis L, Vekrellis K (2010) Cell-produced $\alpha$-synuclein is secreted in a calcium-dependent manner by exosomes and impacts neuronal survival. J Neurosci 30:6838-6851.

Farah CA, Perreault S, Liazoghli D, Desjardins M, Anton A, Lauzon M, Paiement J, Leclerc N (2006) Tau interacts with Golgi membranes and mediates their association with microtubules. Cell Motil Cytoskeleton 63:710-724.

Frost B, Diamond MI (2010) Prion-like mechanisms in neurodegenerative diseases. Nat Rev Neurosci 11:155-159.

Frost B, Jacks RL, Diamond MI (2009) Propagation of tau misfolding from the outside to the inside of a cell. J Biol Chem 284:12845-12852.

Goedert M, Jakes R (1990) Expression of separate isoforms of human tau protein: correlation with the tau pattern in brain and effects on tubulin polymerization. EMBO J 9:4225-4230.

Gómez-Ramos A, Díaz-Hernández M, Cuadros R, Hernández F, Avila J (2006) Extracellular tau is toxic to neuronal cells. FEBS Lett 580:4842-4850

Gómez-Ramos A, Díaz-Hernández M, Rubio A, Miras-Portugal MT, Avila J 
(2008) Extracellular tau promotes intracellular calcium increase through M1 and M3 muscarinic receptors in neuronal cells. Mol Cell Neurosci 37:673-681.

Grossman M, Farmer J, Leight S, Work M, Moore P, Van Deerlin V, Pratico D, Clark CM, Coslett HB, Chatterjee A, Gee J, Trojanowski JQ, Lee VM (2005) Cerebrospinal fluid profile in frontotemporal dementia and Alzheimer's disease. Ann Neurol 57:721-729.

Hesse C, Rosengren L, Andreasen N, Davidsson P, Vanderstichele H, Vanmechelen E, Blennow K (2001) Transient increase in total tau but not phospho-tau in human cerebrospinal fluid after acute stroke. Neurosci Lett 297:187-190.

Hoover BR, Reed MN, Su J, Penrod RD, Kotilinek LA, Grant MK, Pitstick R, Carlson GA, Lanier LM, Yuan LL, Ashe KH, Liao D (2010) Tau mislocalization to dendritic spines mediates synaptic dysfunction independently of neurodegeneration. Neuron 68:1067-1081.

Ittner LM, Ke YD, Delerue F, Bi M, Gladbach A, van Eersel J, Wölfing H, Chieng BC, Christie MJ, Napier IA, Eckert A, Staufenbiel M, Hardeman E, Götz J (2010) Dendritic function of tau mediates amyloid-beta toxicity in Alzheimer's disease mouse models. Cell 142:387-397.

Jung D, Filliol D, Miehe M, Rendon A (1993) Interaction of brain mitochondria with microtubules reconstituted from brain tubulin and MAP2 or TAU. Cell Motil Cytoskeleton 24:245-255.

Kawarabayashi T, Younkin LH, Saido TC, Shoji M, Ashe KH, Younkin SG (2001) Age-dependent changes in brain, CSF, and plasma amyloid $\beta$ protein in the Tg2576 transgenic mouse model of Alzheimer's disease. J Neurosci 21:372-381.

Kim J, Castellano JM, Jiang H, Basak JM, Parsadanian M, Pham V, Mason SM, Paul SM, Holtzman DM (2009) Overexpression of low-density lipoprotein receptor in the brain markedly inhibits amyloid deposition and increases extracellular A beta clearance. Neuron 64:632-644.

Kim W, Lee S, Jung C, Ahmed A, Lee G, Hall GF (2010a) Interneuronal transfer of human tau between Lamprey central neurons in situ. J Alzheimers Dis 19:647-664.

Kim W, Lee S, Hall GF (2010b) Secretion of human tau fragments resembling CSF-tau in Alzheimer's disease is modulated by the presence of the exon 2 insert. FEBS Lett 584:3085-3088.

Lee VM, Goedert M, Trojanowski JQ (2001) Neurodegenerative tauopathies. Annu Rev Neurosci 24:1121-1159.

LoPresti P, Szuchet S, Papasozomenos SC, Zinkowski RP, Binder LI (1995) Functional implications for the microtubule-associated protein tau: localization in oligodendrocytes. Proc Natl Acad Sci U S A 92:10369-10373.

Menacherry S, Hubert W, Justice JB Jr (1992) In vivo calibration of microdialysis probes for exogenous compounds. Anal Chem 64:577-583.

Mocanu MM, Nissen A, Eckermann K, Khlistunova I, Biernat J, Drexler D, Petrova O, Schönig K, Bujard H, Mandelkow E, Zhou L, Rune G, Mandelkow EM (2008) The potential for $\beta$-structure in the repeat domain of tau protein determines aggregation, synaptic decay, neuronal loss, and coassembly with endogenous Tau in inducible mouse models of tauopathy. J Neurosci 28:737-748.

Nickel W (2010) Pathways of unconventional protein secretion. Curr Opin Biotechnol 21:621-626.

Oddo S, Vasilevko V, Caccamo A, Kitazawa M, Cribbs DH, LaFerla FM (2006) Reduction of soluble Abeta and tau, but not soluble Abeta alone, ameliorates cognitive decline in transgenic mice with plaques and tangles. J Biol Chem 281:39413-39423.

Otto M, Wiltfang J, Tumani H, Zerr I, Lantsch M, Kornhuber J, Weber T, Kretzschmar HA, Poser S (1997) Elevated levels of tau-protein in cerebrospinal fluid of patients with Creutzfeldt-Jakob disease. Neurosci Lett 225:210-212.

Perrin RJ, Fagan AM, Holtzman DM (2009) Multimodal techniques for diagnosis and prognosis of Alzheimer's disease. Nature 461:916-922.

Polydoro M, Acker CM, Duff K, Castillo PE, Davies P (2009) Age- dependent impairment of cognitive and synaptic function in the htau mouse model of tau pathology. J Neurosci 29:10741-10749.

Portelius E, Hansson SF, Tran AJ, Zetterberg H, Grognet P, Vanmechelen E, Höglund K, Brinkmalm G, Westman-Brinkmalm A, Nordhoff E, Blennow K, Gobom J (2008) Characterization of tau in cerebrospinal fluid using mass spectrometry. J Proteome Res 7:2114-2120.

Porzig R, Singer D, Hoffmann R (2007) Epitope mapping of mAbs AT8 and Tau5 directed against hyperphosphorylated regions of the human tau protein. Biochem Biophys Res Commun 358:644-649.

Riemenschneider M, Wagenpfeil S, Vanderstichele H, Otto M, Wiltfang J, Kretzschmar H, Vanmechelen E, Förstl H, Kurz A (2003) Phospho-tau/ total tau ratio in cerebrospinal fluid discriminates Creutzfeldt-Jakob disease from other dementias. Mol Psychiatry 8:343-347.

Roberson ED, Scearce-Levie K, Palop JJ, Yan F, Cheng IH, Wu T, Gerstein H, Yu GQ, Mucke L (2007) Reducing endogenous tau ameliorates amyloid beta-induced deficits in an Alzheimer's disease mouse model. Science 316:750-754

Roberson ED, Halabisky B, Yoo JW, Yao J, Chin J, Yan F, Wu T, Hamto P, Devidze N, Yu GQ, Palop JJ, Noebels JL, Mucke L (2011) Amyloid- $\beta$ / Fyn-induced synaptic, network, and cognitive impairments depend on tau levels in multiple mouse models of Alzheimer's disease. J Neurosci 31:700-711.

Santacruz K, Lewis J, Spires T, Paulson J, Kotilinek L, Ingelsson M, Guimaraes A, DeTure M, Ramsden M, McGowan E, Forster C, Yue M, Orne J, Janus C, Mariash A, Kuskowski M, Hyman B, Hutton M, Ashe KH (2005) Tau suppression in a neurodegenerative mouse model improves memory function. Science 309:476-481.

Sigurdsson EM (2009) Tau-focused immunotherapy for Alzheimer's disease and related tauopathies. Curr Alzheimer Res 6:446-450.

Small SA, Duff K (2008) Linking Abeta and tau in late-onset Alzheimer's disease: a dual pathway hypothesis. Neuron 60:534-542.

Sydow A, Mandelkow EM (2010) 'Prion-like' propagation of mouse and human tau aggregates in an inducible mouse model of tauopathy. Neurodegener Dis 7:28-31.

Sydow A, Van der Jeugd A, Zheng F, Ahmed T, Balschun D, Petrova O, Drexler D, Zhou L, Rune G, Mandelkow E, D’Hooge R, Alzheimer C, Mandelkow EM (2011) Tau-induced defects in synaptic plasticity, learning, and memory are reversible in transgenic mice after switching off the toxic Tau mutant. J Neurosci 31:2511-2525.

Takuma H, Arawaka S, Mori H (2003) Isoforms changes of tau protein during development in various species. Brain Res Dev Brain Res 142:121-127.

Thies E, Mandelkow EM (2007) Missorting of tau in neurons causes degeneration of synapses that can be rescued by the kinase MARK2/Par-1. J Neurosci 27:2896-2907.

Trickler WJ, Miller DW (2003) Use of osmotic agents in microdialysis studies to improve the recovery of macromolecules. J Pharm Sci 92:1419-1427.

Vandermeeren M, Mercken M, Vanmechelen E, Six J, van de Voorde A, Martin JJ, Cras P (1993) Detection of tau proteins in normal and Alzheimer's disease cerebrospinal fluid with a sensitive sandwich enzymelinked immunosorbent assay. J Neurochem 61:1828-1834.

von Bergen M, Friedhoff P, Biernat J, Heberle J, Mandelkow EM, Mandelkow E (2000) Assembly of tau protein into Alzheimer paired helical filaments depends on a local sequence motif ((306)VQIVYK(311)) forming beta structure. Proc Natl Acad Sci U S A 97:5129-5134.

Weller RO, Subash M, Preston SD, Mazanti I, Carare RO (2008) Perivascular drainage of amyloid-beta peptides from the brain and its failure in cerebral amyloid angiopathy and Alzheimer's disease. Brain Pathol 18:253-266.

Yoshiyama Y, Higuchi M, Zhang B, Huang SM, Iwata N, Saido TC, Maeda J, Suhara T, Trojanowski JQ, Lee VM (2007) Synapse loss and microglial activation precede tangles in a P301S tauopathy mouse model. Neuron $53: 337-351$ 